\title{
Guias alimentares para a população brasileira: uma análise a partir das dimensões culturais e sociais da alimentação
}

\author{
Dietary guidelines for Brazilian population: an analysis \\ from the cultural and social dimensions of food
}

Mayara Sanay da Silva Oliveira (https://orcid.org/0000-0002-3243-2575) ${ }^{1}$

Ligia Amparo da Silva Santos (https://orcid.org/0000-0002-6925-6421) ${ }^{2}$

\footnotetext{
${ }^{1}$ Programa de PósGraduação em Alimentos, Nutrição e Saúde, Escola de Nutrição, Universidade Federal da Bahia (UFBA). Av. Araújo Pinho 32, Canela. 40110-150 Salvador BA Brasil. mayarasanay@gmail.com ${ }^{2}$ Departamento de Ciência da Nutrição, Escola de Nutrição, UFBA. Salvador BA Brasil.
}

\begin{abstract}
This article analyzes the social and cultural dimensions of food addressed in the dietary guidelines of the second edition of the Dietary Guideline for the Brazilian Population (DGBP) in contrast to its first edition. This is a qualitative study. We employ the Pecheutian discourse analysis. The study universe is the first and second edition of DGBP. Data analysis used three steps: identification of similar and different texts of these materials; elucidation of the social and cultural dimensions of food in these materials; and systematic analysis with contrasting emerging discourses in both DGBPs. We emphasize that in the second edition the theoretical references-epidemiological, clinical, sociological, anthropological studies, and popular knowledge - and the use of the NOVA food classification favored the development of more holistic dietary guidelines that address food and eating patterns, culinary practices, the act of eating and the commensality. It is concluded that the second editions of DGPB allows health professionals and the population an understanding of food as something concrete in the life of individuals and collectivity, extrapolating its physiological and biological dimension.
\end{abstract}

Key words Food-based dietary guidelines, Healthy eating, Cooking, Commensality, Traditional food
Resumo Este artigo analisa as dimensões sociais e culturais da alimentação abordadas nas diretrizes alimentares da segunda edição do Guia Alimentar para a População Brasileira (GAPB), contrapondo-o com a sua primeira edição. Tratase de um estudo qualitativo. Empregamos a análise de discurso Pecheutiana. O universo de estudo são a primeira e a segunda edição do GAPB. A análise dos dados utilizou três etapas: identificação de textos similares e diferentes dos materiais; elucidação das dimensões sociais e culturais da alimentação nos materiais; e análise sistemática com contraposição dos discursos emergentes em ambos os GAPBs. Enfatizamos que na segunda edição os referenciais teóricos - estudos epidemiológicos, clínicos, sociológicos, antropológicos e saberes populares - e a utilização da classificação de alimentos NOVA favoreceram o desenvolvimento de diretrizes alimentares mais holísticas que abordam os padrões de alimentação e refeição, as práticas culinárias, o ato de comer e a comensalidade. Conclui-se que a segunda edição do GAPB possibilita aos profissionais de saúde e à população uma compreensão do alimento enquanto parte concreta da vida dos indivíduos e coletividade, extrapolando sua dimensão fisiológica e biológica. Palavras-chave Guias alimentares, Alimentação saudável, Culinária, Comensalidade, Alimentação tradicional 


\section{Introdução}

Os Guias Alimentares Baseados em Alimentos (GABA) são definidos como instrumentos que expressam os princípios da alimentação e da nutrição através de mensagens práticas para educar a população e guiar as políticas públicas de alimentação e nutrição, saúde e agricultura ${ }^{1}$. No Brasil, a experiência com GABA é concretizada em duas edições do Guia Alimentar para a População Brasileira (GAPB), publicados nos anos $2006^{2}$ e $2014^{3}$. Os GAPBs visam proporcionar orientações para a realização de práticas alimentares apropriadas aos aspectos biológicos, socioculturais e ao uso do meio ambiente sustentável, com vistas à prevenção das Doenças Crônicas Não Transmissíveis (DCNT) e diminuição das comorbidades associadas ${ }^{4}$.

Em análise comparativa dos GAPB 2006 e 2014, Oliveira e Silva-Amparo ${ }^{5}$ ressaltaram que a adoção da classificação NOVA possibilitou o desenvolvimento de diretrizes alimentares atentas às limitações do discurso nutricional e que incorporam argumentações socioculturais e socioambientais, evocando o prazer na alimentação, a diversidade de hábitos alimentares e o estimulo à prática culinária para a Promoção da Alimentação Saudável e Adequada (PAAS). Monteiro et al. ${ }^{6}$ denotam que as diretrizes alimentares da segunda edição identificam as dimensões biológicas, comportamentais, culturais, socioeconômicas e ambientais da alimentação no contexto de padrões alimentares baseados em alimentos in natura ou minimamente processados. Por sua vez, Davies et al. ${ }^{7}$ consideram que a segunda edição do GAPB inova ao dar mais valor ao contexto de consumo alimentar, aos valores socioculturais do ato de comer, às diferentes formas de conhecimento e à autonomia dos indivíduos.

Dentre os artigos supracitados, dois utilizaram métodos científicos qualitativos ${ }^{5,7}$ e um realizou análise comparativa dos GAPB 2006 e 20147. Apesar dos artigos expressarem a importância das dimensões sociais e culturais dos $\mathrm{GAPBs}^{5-7}$, nenhum aprofundou a discussão dessa temática. Assim, este artigo aborda a lacuna de literatura de estudos qualitativos e comparativos das dimensões sociais e culturais da alimentação expressas nas diretrizes alimentares dos GAPBs. Objetiva-se analisar as dimensões sociais e culturais da alimentação abordadas nas diretrizes alimentares da segunda edição do GAPB, contrapondo-o com a sua primeira edição.

\section{Métodos}

Conduziu-se um estudo qualitativo utilizando análise do discurso Pechêutiana na perspectiva de compreender as similaridade e as disparidades relacionadas aos discursos das dimensões sociais e culturais da alimentação nos GAPBs. O universo da pesquisa são a primeira e a segunda edição do GAPB (doravante GAPB 2006 e GAPB 2014). O corpus foi estabelecido por meio de: leitura flutuante examinando o índice, as imagens, os textos escritos nos dois GAPBs; leitura aprofundada caracterizada pela busca de temas ou assuntos nos índices, imagens e textos escritos com identificação das sequencias discursivas que expressem as dimensões sociais e culturais da alimentação; seleção das sequências discursivas por dois pesquisadores com experiência em pesquisa qualitativa; e, organização das sequências discursivas em uma matriz para análise com explicitação do título do documento, capítulo, página, sequência discursiva e convergências e divergências entre os discursos $^{8}$.

A análise dos dados seguiu os passos propostos por Pêcheux': identificação de textos semelhantes que continham uma equivalência discursiva aos GAPB 2014 e 2006; elucidação das sequencias discursivas que expressassem os discursos sociais e culturais da alimentação; análise sistemática das sequências discursivas que expressam equivalência ou disparidade entre os GAPB 2006 e 2014. Por fim, foi obtida uma matriz relacionada ao contexto de produção dos materiais, aos princípios dos GAPB, à classificação dos alimentos utilizados e às orientações dietéticas presentes nos materiais.

\section{Resultados e discussão}

\section{Referenciais teóricos}

No GAPB 2006, os principais referenciais teóricos eram os estudos epidemiológicos e clínicos que buscavam relacionar os alimentos, grupos alimentares e nutrientes na redução ou aumento do risco de ocorrência de DCNT. Fardet e Rock ${ }^{10}$ sugerem que a utilização referencial teórico dessa natureza podem gerar diretrizes alimentares mais focadas nos nutrientes e em outros componentes dos alimentos, em detrimento dos alimentos e das refeições tradicionais que são dotados de significados históricos, sociais e culturais que influenciam nos comportamentos alimentares. 
O GAPB 2014 incorporou os estudos sociológicos e antropológicos e os saberes populares aos estudos epidemiológicos e clínicos em seus referenciais teóricos. A incorporação de referenciais teóricos das ciências humanas representa uma tentativa de abarcar o caráter multidimensional da alimentação, a complexidade da vida dos indivíduos e sua interação na sociedade ${ }^{11}$. Tal ampliação pode ser percebida nas fotografias do GAPB 2014 que apresentam a alimentação de maneiras mais realista através do ato de comer, dos grupos sociais, das relações familiares, da comensalidade e dos alimentos locais e regionais (por exemplo, tapioca, cuscuz, polenta, feijão, feijoada).

\section{Os sistemas de classificações de alimentos}

No GAPB 2006, o sistema de classificação de alimentos era similar à pirâmide alimentar estadunidense, porém adaptada à realidade brasileira. Nele, os alimentos eram organizados em grupos alimentares de acordo com suas características nutricionais ou biológicas ${ }^{12}$. Os grupos alimentares eram cereais, raízes e tubérculos; frutas, legumes e verduras; leite e seus derivados; carne e ovos; feijões e outros alimentos ricos em proteínas; óleos e gorduras; e açúcares e doces. Ainda, esse material utiliza o conceito de porções alimentares que corresponde à quantidade média de alimentos que devem ser consumidos em termos de energia e nutrientes por um indivíduo saudável ${ }^{13}$. Ambos os conceitos embasavam as diretrizes dietéticas que incentivam uma alimentação saudável constituídas por três tipos de alimentos básicos:

1) alimentos com alta concentração de carboidratos, como grãos, pães, massas, tubérculos e raízes; 2) Frutas, legumes e verduras; e 3) Alimentos vegetais ricos em proteinas (particularmente os cereais integrais, as leguminosas e as sementes e as castanhas $)^{2}$.

Percebe-se que a abordagem da alimentação saudável no GAPB 2006 está focada nos nutrientes e nos componentes bioquímicos dos alimentos, desconectados da complexidade da vida cotidiana, dos ambientes alimentares e do modelo de saúde mais abrangente ${ }^{5}$.

Por sua vez, o GAPB 2014 adotou a classificação de alimentos NOVA baseada na natureza, extensão e propósito do processamento de alimentos antes da aquisição, preparação e consumo do alimento ${ }^{6}$. Nessa classificação, os alimentos são agrupados em: in natura ou minimamente processados; ingredientes culinários; processados; e ultraprocessados ${ }^{6}$. O material não utiliza o conceito de porção alimentar ou qualquer ou- tra forma de quantificação. O padrão alimentar, então, advém do consumo de uma variedade de alimentos in natura e minimamente processados, contextualizado nas refeições tradicionais e preparações culinárias brasileiras.

Os alimentos in natura e os minimamente processados são valorizados em resposta ao impacto aumento do consumo dos ultraprocessados nos perfis de morbimortalidade brasileiro. No período de 2000 a 2013, as vendas de alimentos ultraprocessados aumentaram em $30,6 \%{ }^{14}$. Em 2008-2009, a Pesquisa de Orçamentos Familiares-POF registrou a diminuição no consumo de alimentos tradicionais brasileiros, refletidos no arroz, feijão e mandioca. Em contrapartida, expressou o aumento do consumo de alimentos ultraprocessados, como os biscoitos, embutidos, refrigerantes e refeições prontas e misturas industrializadas ${ }^{15}$. De acordo com Louzada et al. ${ }^{16}$, 21,5\% do consumo médio de energia diário dos brasileiros advém de alimentos ultraprocessados. De outra parte, crescentes evidências científicas associam o aumento do consumo de alimentos ultraprocessados ao aumento da obesidade e DCNT no Brasil ${ }^{17,18}$. Em contraposição, estudos mostram que uma dieta tradicional, baseada em alimentos in natura ou minimamente processados, apresentam significativos benefícios à saúde e são protetoras contra as $\mathrm{DCNT}^{19,20}$.

\section{Os princípios das diretrizes alimentares}

No GAPB 2006, os princípios consolidavam diretrizes nutricionais voltadas para a prevenção de doenças, alcançada pelo consumo quantitativo de grupos de alimentos:

abordagem integrada; referencial científico e a cultura alimentar; referencial positivo; explicitação de quantidades; variações das quantidades; alimento como referência; sustentabilidade ambiental; originalidade: um guia Brasileiro; abordagem multifocal ${ }^{2}$.

No GAPB 2014, a ideia central é encorajar o consumo alimentos in natura ou minimamente processados, preparações culinárias e refeições baseadas nestes alimentos. Para tanto, o material utiliza os seguintes princípios:

A alimentação é mais que a ingestão de nutrientes; recomendações sobre alimentação devem estar em sintonia com seu tempo; alimentação adequada e saudável deriva de sistemas alimentar socialmente e ambientalmente sustentável; diferentes saberes geram o conhecimento para a formulação de guias alimentares; guias alimentares ampliam a autonomia nas escolhas alimentares ${ }^{3}$. 
Percebe-se que ambos os GAPBs consideram que os alimentos não apenas veiculam nutrientes. Contudo, o GAPB 2014 acrescenta que os alimentos veiculam compostos com atividade biológica e potenciais para a saúde ${ }^{21}$, e valores simbólicos e emocionais desenvolvidos no meio sociocultural ${ }^{22}$.

O GAPB 2014 utiliza a classificação NOVA e a regra de ouro "prefira sempre alimentos in natura ou minimamente processados e preparações culinárias a alimentos ultraprocessados" que permitiram a reconfiguração do valor atribuído à culinária, às práticas alimentares, aos padrões alimentares tradicionais, ao ato de comer e à comensalidade, ao mesmo tempo em que apresenta estratégias para PAAS.

\section{Os padrões alimentares tradicionais dos brasileiros}

O GAPB 2006 apresentava recomendações que visavam promover o resgate dos hábitos alimentares regionais inerentes ao consumo de alimentos produzidos localmente e culturalmente referenciados - enquanto estratégia para promoção e consolidação dos padrões alimentares regionais.

O enfoque assumido neste guia, com o claro incentivo ao consumo de alimentos nas formas mais naturais e produzidos localmente e à valorização dos alimentos regionais e da produção familiar e da cultura alimentar, além de estimular mudanças de hábitos alimentares para a redução do risco de ocorrência de doenças, valoriza a produção e o processamento de alimentos com o uso de recursose tecnologias ambientalmente sustentáveis ${ }^{2}$.

No GAPB 2014, apenas nele, os padrões tradicionais de alimentação estão relacionados com a identidade e o sentimento de pertencimento social e cultural, com a sensação de autonomia, com o prazer propiciado pela alimentação e, consequentemente, com o estado de bem-estar dos indivíduos. Os padrões alimentares e os modos de comer marcam o engajamento dos indivíduos em certos grupos sociais determinados por condições étnicas, socioeconômicas e outras, da mesma forma expressa as identidades das pessoas ${ }^{22}$.

Considera-se, também, que os padrões alimentares tradicionais apresentam efeitos positivos à saúde, diante da interação entre os componentes dos alimentos, das preparações culinárias ou da refeição.

Alimentos específicos, preparações culinárias que resultam da combinação e preparo desses alimentos e modos de comer particulares constituem parte importante da cultura de uma sociedade e, como tal, estão fortemente relacionados com a identidade e o sentimento de pertencimento social das pessoas com a sensação de autonomia, com o prazer propiciado pela alimentação e, consequentemente, com o seu estado de bem-estar ${ }^{3}$.

O princípio "recomendações sobre alimentação devem estar em sintonia com seu tempo" proporcionou o desenvolvimento de diretrizes alimentares que pensam o comer contemporâneo em suas particularidades. $\mathrm{O}$ comer contemporâneo é marcado pela desvalorização do preparo e consumo de refeições caseiras e tradicionais, flexibilização dos horários das refeições e individualização dos rituais alimentares ${ }^{23}$. Nesse cenário, os sistemas normativos e de controle social que tradicionalmente regiam as práticas e as representações alimentares são enfraquecidas ${ }^{24}$; e o setor saúde considera o retorno às práticas alimentares tradicionais ${ }^{25}$ como estratégia para a regeneração dos sistemas alimentares locais e o aumento da sustentabilidade social das práticas alimentares $^{26}$.

\section{Habilidades e prática culinária}

O GAPB 2006 valorizava a culinária nacional ressaltando as técnicas culinárias como elementos determinantes da qualidade nutricional dos alimentos e a importância das preparações caseiras para efetivação de práticas alimentares saudáveis.

Desenvolver ações de valorização da culinária nacional que promovam o consumo de preparações e alimentos saudáveis $[\ldots]^{2}$.

No GAPB 2006, a culinária nacional era vista como uma coleção de receitas com ingredientes diversos que compunham pratos que poderiam ser saudáveis e promotores de saúde ou o contrário. O material se mostrava preocupado com o consumo excessivo de gorduras, açúcares e sal através de preparações culinárias típicas.

A alimentação com alto teor de sal e alimentos salgados, como muitas das preparações típicas da culinária Brasileira, aumentam o risco de hipertensão arterial, acidentes vasculares e câncer de estômago ${ }^{2}$.

Por consequência, recomendava ponderação em relação a algumas preparações culinárias tradicionais, denotando que as técnicas de preparo e os ingredientes utilizados poderiam interferir na qualidade nutricional das preparações culinárias, tornando-as promotoras de enfermidades e inadequadas à alimentação saudável, por exemplo: a feijoada ${ }^{27}$. 
Feijoada e outros pratos feitos com feijão e carnes gordas, embutidos, toucinho e outros tipos de carnes têm alto teor de gordura saturada e de sal, o que não é saudável; consuma esse tipo de preparação ocasionalmente ${ }^{2}$.

No GAPB 2014, a prática culinária é uma estratégia para diminuição do consumo de alimentos ultraprocessados, e a culinária nacional é considerada um processo sociocultural relacionados com a identidade e o sentimento de pertencimento social e cultural das pessoas, a autonomia, o prazer e o bem-estar dos indivíduos e das coletividades ${ }^{27}$.

Em contraste com alimentos ultraprocessados, alimentos in natura ou minimamente processados usualmente precisam ser selecionados, pré-preparados, temperados, cozidos, combinados a outros alimentos e apresentados na forma de pratos para que possam ser consumidos. As habilidades envolvidas com seleção, pré-preparo, tempero, cozimento, combinação e apresentação dos alimentos são as habilidades culinárias. Dessas habilidades, desenvolvidas em cada sociedade e aperfeiçoadas e transmitidas ao longo de gerações, dependem o sabor, o aroma, a textura e a aparência que os alimentos in natura ou minimamente processados irão adquirir e o quanto eles serão apreciados pelas pessoas ${ }^{3}$.

Estudos demonstram que as habilidades culinárias e o prazer/gosto por cozinhar apresentam impactos na diminuição do consumo de alimentos ultraprocessados, no aumento do consumo de frutas e vegetais, na redução do risco de excesso de peso e obesidade ${ }^{28,29}$. Contudo, a culinária passa por um processo de "transição" caracterizado por mudanças nos padrões e nas habilidades necessárias para obter, preparar e consumir os alimentos ${ }^{30}$. Dados da POF 2008-2009 revelam que os brasileiros gastam $31 \%$ do orçamento familiar com alimentação fora de casa, representando um aumento quando comparado ao período de 2002-2003, cujos gastos eram equivalentes a $24 \%$ do orçamento ${ }^{15}$. Um estudo com 1.502 adultos, encontrou que $25 \%$ dos entrevistados cozinhavam diariamente ou na maioria dos dias da semana, dedicando em média 5,2 horas semanais a essa atividade ${ }^{31}$. Os estudos apresentados sugerem uma baixa adesão à prática culinária e a expansão da alimentação fora de casa.

Nesse cenário, o GAPB 2014 considera a prática culinária enquanto elemento a ser promovido e preservado, não se limitando apenas ao seu impacto na qualidade nutricional da alimentação, ou no perfil de morbidade da população. Mas, por sua dimensão antropológica que inclui um sistema ideológico, simbólico, de normas e crenças que norteia a sua prática, expressos na história, na tradição, nas tecnologias, nos procedimentos, nos ingredientes e nos atores, bem como nas identidades territoriais, de gênero, de classe e outras que conformam a culinária doméstica $^{27,32}$. Por consequência, O GAPB 2014 incentiva as pessoas a adquirirem, desenvolverem e partilharem habilidades culinárias com outras pessoas em seus grupos sociais.

Se você tem habilidades culinárias, procure desenvolvê-las e partilhá-las, principalmente com crianças e jovens, sem distinção de gênero. Se você não tem habilidades culinárias - e isso vale para homens e mulheres -, procure adquiri-las. Para isso, converse com as pessoas que sabem cozinhar, peça receitas a familiares, amigos e colegas, leia livros, consulte a internet, eventualmente faça cursos e comece a cozinhar! ${ }^{3}$.

Além disso, o GAPB 2014 evoca o prazer presente no ato de preparar, combinar e cozinhar alimentos e de compartilhar as habilidades culinárias com outras pessoas. Reconhecendo de maneira positiva as práticas culinárias, reforçando a autonomia das pessoas no preparo do próprio alimento, e uma maior compreensão e reflexão das dimensões sensoriais, cognitivas e simbólicas da alimentação.

Contudo, dentre outros fatores, a divisão do trabalho culinário doméstico compreende um limitante importante para o desenvolvimento da culinária $^{29}$. Em ambos os GAPBs, o ingresso da mulher no mercado de trabalho e as dificuldades de mudanças nos papeis tradicionais de gênero são discriminados enquanto um dos elementos de enfraquecimento do processo de transmissão geracional das habilidades culinárias e da desvalorização da culinária doméstica.

[...] quando a mulher assume uma vida profissional extradomicílio, continua ainda acumulando a responsabilidade sobre a alimentação da família. A atribuição de atividades à mulher no ambiente do trabalho remunerado e no espaço doméstico se coloca como um novo paradigma da sociedade moderna, que não tem criado mecanismos de suporte social para a desconcentração dessa atribuição como exclusivamente feminina ${ }^{2}$.

No Brasil, e em muitos outros países, o processo de transmissão de habilidades culinárias entre gerações vem perdendo força e as pessoas mais jovens possuem cada vez menos confiança e autonomia para preparar alimentos. As razões para isso são complexas envolvem a desvalorização do ato de preparar, combinar e cozinhar alimentos como prática cultural e social, a multiplicação das tarefas cotidianas e a incorporação da mulher no mercado 
formal de trabalho, além da oferta massiva e da publicidade agressiva dos alimentos ultraprocessa$\operatorname{dos}[\ldots]^{3}$.

Para atuar sobre a situação, o GAPB 2006 sugere a criação de mecanismos de suporte social para a desconcentração dessa atribuição como exclusivamente feminina e o GAPB 2014 recomenda o compartilhamento das habilidades culinárias sem distinção de gênero, e o desenvolvimento da prática culinária por todos os membros da família de forma a não concentrar a responsabilidade em uma única pessoa.

Contudo, estudos nacionais têm destacado a centralidade das mulheres brasileiras - especialmente das mães - na responsabilidade pela compra, preparo e oferecimento das comidas para a família $^{31,33,34}$ e dos homens na culinária festiva e na oferta de recursos para aquisição dos alimen$\operatorname{tos}^{35}$. O GAPB 2014 promove a discussão da divisão do trabalho doméstico ao sugerir ações no plano pessoal e familiar para a desconcentração da prática culinária das mulheres. Porém, essas recomendações devem ser articuladas com estratégias sociopolíticas e socioeconômicas que favoreçam a redução da sobrecarga com as responsabilidades em relação ao trabalho doméstico pelas mulheres. A FAO sugere medidas relacionadas à melhoria dos serviços de transporte público, água e energia, serviços de assistência à infância e cuidados institucionais para os doentes e idosos e a licença parental tanto para as mulheres quanto para os homens ${ }^{36}$.

Além da divisão do trabalho culinário doméstico, o GAPB 2014 apresenta o tempo enquanto obstáculo para a prática culinária. Nesse documento, apenas nele, as crescentes demandas do mundo profissional e social são apresentadas enquanto limitante do tempo destinado para a prática culinária. $\mathrm{O}$ documento propõe uma:

[...] revisão crítica do uso do tempo de cada pessoa não deve ser entendida como indicação de que a falta de tempo não chega a ser um problema ou é problema cuja solução demanda apenas ações no plano individual. Como no caso dos demais obstáculos, o que este guia propõe para seus leitores é uma combinação de ações no plano pessoal e familiar e ações no plano da cidadania. A atuação no plano coletivo, neste caso, seria exemplificada pela defesa de políticas públicas eficazes para diminuir o tempo que as pessoas gastam no seu deslocamento, como o investimento no transporte público e o uso mais racional das vias de transporte ${ }^{3}$.

Estudos sugerem que as pessoas convivem com um sentimento crônico de falta de tempo, destinando menor tempo à alimentação e à prá- tica culinária doméstica, e maior para a produtividade no trabalho ${ }^{37}$. Como resultado, as pessoas tendem a considerar desperdício de tempo e esforço o preparo de alimentos no espaço doméstico $^{38}$, substituindo as refeições tradicionais por alimentos prontos para consumo ${ }^{39}$. Soma-se a esse cenário, o processo de urbanização que promoveu a externalização dos processos culinários, diminuindo a importância do preparo caseiro de alimentos e profissionalizando a produção de alimentos ${ }^{40}$. Assim, as pessoas podem considerar o cozinhar enquanto disponibilização de tempo para outra pessoa, sendo mais valorizada quando destinada à família ou a outras pessoas dos grupos sociais, e menos quando o preparo da comida é destinado para si, e apresenta o final de semana e a data festiva como principais momentos para o seu desenvolvimento ${ }^{40}$.

Ciente de que a prática culinária durante os dias úteis da semana compreende um obstáculo a ser superado, o GAPB 2014 destaca a adesão aos Programas Nacionais de Alimentação do Trabalhador (PAT) e de Alimentação Escolar (PNAE) enquanto protetores das práticas alimentares saudáveis, pois ambos visam o incentivo ao consumo de preparações culinárias localmente referenciadas e à diminuição do consumo de alimentos ultraprocessados. Segundo Azeredo et al. ${ }^{41}, \mathrm{o}$ consumo dos alimentos das refeições oferecidas pelo PNAE está associado com a menor chance de consumo regular de refrigerantes, salgados fritos, salgadinhos ensacados e doces. Sendo assim, as diretrizes do GAPB 2014 incentivam o controle social e a melhoria, a execução e a fiscalização desses programas. Ressalta-se a necessidade de instigar o consumo das refeições oferecidas nos diferentes programas institucionais de alimentação e nutrição visto que compreendem estratégias concretas para a diminuição do consumo de alimentos ultraprocessados ${ }^{41}$.

\section{$O$ ato de comer e a comensalidade}

O GAPB 2006 buscou revalorizar as refeições em grupo enquanto resposta ao isolamento dos grupos familiares e o crescente número de refeições feitas pelo indivíduo de maneira solitária, mesmo quanto coabitando um mesmo lar.

Nas sociedades modernas, as pessoas cada vez mais se isolam dos outros membros da família, mesmo quando estão sob o mesmo teto. É crescente o número de refeições feitas pelo indivíduo de maneira solitária, fora de casa e mesmo em casa. Não é esta a proposta deste guia. Ao contrário, valori$z a$-se o ato de alimentar-se no ambiente familiar, 
permitindo a integração das pessoas por meio do compartilhar o momento da alimentação, como importante para o fortalecimento das relações afetivas e de integração familiar ${ }^{2}$.

No GAPB 2014, o capítulo 04 dedica recomendações sobre o ato de comer e a comensalidade, expressando três diretrizes básicas: o comer com regularidade e com atenção, o comer em ambientes apropriados e o comer em companhia. Em suma, todas as orientações visam restabelecer os sistemas normativos e de controle social que regem as práticas e as representações alimentares $^{24}$, visto que esses são considerados protetores contra o consumo de alimentos ultraprocessa$\operatorname{dos}^{42}$.

Através destas três diretrizes, o GAPB 2014 valoriza a realização de refeições tradicionais dos brasileiros, em seus horários, locais e companhias determinados socioculturalmente, e produzidas por meio de preparações culinárias caseiras. Essas recomendações são justificadas pela diminuição paulatina da realização das refeições tradicionais em horários regulares do cotidiano das pessoas; e a expressa substituição do consumo das refeições tradicionais por alimentos ultraprocessados que podem ser ingeridos sem cutelaria, durante a realização de outras atividades (estudo/trabalho) e em qualquer espaço (mesa de trabalho, meio de transporte, entre outros) ${ }^{16,43}$. Nesse cenário, as práticas alimentares contemporâneas passam por uma crise de seus sistemas normativos e de controle social ${ }^{25}$; ou seja, do conjunto de orientações sobre condutas mais ou menos apropriadas para a alimentação/modos de comer dentro de um contexto particular, determinada diante de padrões culturais (recursos tecnológicos, organização social, atividades, horários, profissão, relacionamentos familiares, responsabilidade, entre outros $)^{44}$.

Resgatar a comensalidade valoriza o ato de comer enquanto uma atividade social, contrapondo os modelos individualizantes propulsionados pela indústria de alimentos ultraproces$\operatorname{sados}^{45}$. Nesta crença, o GAPB 2014 alude que o compartilhamento de refeições realizadas em ambientes domésticos pode ser um momento precioso:

[...] para os casais, momentos de encontro para saber um do outro e planejar o futuro da família; para as crianças e adolescentes, o momento é pertinente para criação de hábitos alimentares saudáveis e a valorização das refeições regulares e feitas em ambientes apropriados ${ }^{3}$.
Decerto que o ambiente familiar pode apresentar grande influência nos hábitos alimentares das pessoas, principalmente em crianças e adolescentes, já que disponibiliza aos indivíduos: os alimentos e as preparações culinárias para consumo, os modelos de comportamentos, o apoio para as práticas alimentares e o desenvolvimento das competências alimentares adequadas à saúde ${ }^{46}$. Estudos sugerem que a frequência das refeições em conjunto está associada ao maior consumo de alimentos saudáveis e menor de alimentos ultraprocessados ${ }^{47,48}$, além de afetarem positivamente as emoções, as atitudes e os comportamentos dos indivíduos, a repercussão na satisfação de vida, o conforto psicológico e a união familiar positiva ${ }^{49}$.

Conquanto, a comensalidade nem sempre compreende um momento benéfico, podendo ser momentos de conflito da vida diária, que pode gerar ansiedade sobre o uso simbólico dos alimentos na convivência ${ }^{50,51}$. Em perspectiva sociológica, a comensalidade é uma das expressões mais marcantes da sociabilidade humana e, como tal, não exclui contradições sociais e relações de poder, emaranhando em um processo relacionado com a estrutura social em que se vive ${ }^{45}$. A comensalidade compreende, ainda, um sistema de comunicação inter-relacional em que a disposição dos comensais, a distribuição e a partilha dos alimentos e as "boas maneiras à mesa" expressam o status social, o estilo de vida, o afeto e a dinâmica social da família ${ }^{52}$. Por consequência, as refeições em família podem ser momentos de tensão quando transbordam os conflitos familiares, revelando as relações de opressão que reforçam a estrutura de poder nos ambientes domésti$\cos ^{50,51}$. Outra dimensão das refeições em família se refere ao fato de que: comer junto nem sempre expressa socialização. Em algumas famílias, as refeições são realizadas em espaço comum, contudo em um ritual individualizado que expressa o consumo do alimento em silêncio ${ }^{52}$. Em síntese, os contextos apresentados exprimem que a comensalidade por si só pode não apresentar uma relação direta com o caráter protetor de práticas alimentares saudáveis ou de proteção da saúde, e que nas práticas educativas em saúde, alimentação e nutrição é preciso pensá-las nas suas singularidades. Por consequência, o GAPB 2014 sugere que suas recomendações sejam adaptadas às condições específicas de cada pessoa, desde que se respeite a sua regra de ouro. 


\section{Conclusão}

Nesta análise comparativa, identificou-se que ambos os GAPB concebem que a alimentação deriva de práticas alimentares que apresentam significados sociais e culturais. Os materiais consideram que os alimentos agregam significados culturais, comportamentais e afetivos singulares que não podem ser desprezados nas estratégias de promoção da alimentação adequada e saudável. Contudo, o GAPB 2006 apresenta um discurso médico -nutricional das dimensões sociais e culturais da alimentação, limitando a abordagem do padrão alimentar tradicional, da culinária nacional e da comensalidade enquanto estratégias para melhorar a qualidade nutricional dos alimentos e das refeições consumidas pelas famílias brasileiras.

Por sua vez, o GAPB 2014 concebe que uma alimentação saudável deve ser pautada em alimentos in natura ou minimamente processados e preparações culinárias à base desses alimentos. Além disso, o GAPB 2014 utiliza estudos sociológicos, antropológicos e saberes populares para construir diretrizes alimentares mais holísticas que abordam os modos de comer: com regula- ridade e atenção; em ambientes apropriados; em companhia; alimentos produzidos de maneira sustentável ambientalmente e socialmente; com planejamento do uso do tempo para a alimentação; criticamente quanto a informações, orientações e mensagens sobre alimentos veiculadas em propagandas comerciais; praticando a culinária doméstica; e compartilhando as habilidades e atividades culinárias entre todos os membros da família.

Por fim, o presente artigo apresenta limitações referentes ao aprofundamento de uma discussão epistemológica sobre os modos de incorporação das dimensões socioculturais nos GAPB 2014 e 2006, bem como dos fundamentos teóricos das ciências sociais e humanas nas diretrizes desses materiais. Sugere-se o desenvolvimento de outras pesquisas para estas discussões à luz das ciências sociais e humanas e dos conceitos fundantes do GAPB 2014 (padrão alimentar, práticas alimentares, tradição alimentar, práticas culinárias, comensalidade e outros), pois estes conceitos são intrínsecos ao desenvolvimento de programas e ações para a PAAS dentro das diretrizes da PNAN.

\section{Colaboradores}

Este manuscrito resultou da dissertação de mestrado de MSS Oliveira. L Amparo-Santos forneceu orientação acadêmica para a dissertação e contribuiu substancialmente para melhorar a discussão e os dados analisados.

\section{Agradecimentos}

As autoras agradecem à Profa. Dra. Sandra Maria Chaves dos Santos e a Profa. Dra. Michele Dantas Soares. Apoio financeiro: Coordenação de Aperfeiçoamento de Pessoal de Nível Superior. 


\section{Referências}

1. Food and Agriculture Organization of the United Nations (FAO). Developing Food-Based Dietary Guidelines: a manual from English-speaking Caribbean. Rome: FAO; 2007.

2. Brasil. Ministério da Saúde (MS). Guia alimentar para a População Brasileira: promovendo alimentação saudável. Brasília: MS; 2006.

3. Brasil. Ministério da Saúde (MS). Guia Alimentar para a População Brasileira. Brasília: MS; 2014.

4. Brasil. Ministério da Saúde (MS). Política Nacional de Alimentação e Nutrição. Brasília: MS; 2013.

5. Oliveira MS, Silva-Amparo L. Food-based dietary guidelines: a comparative analysis between Dietary Guidelines for the Brazilian Population 2006 and 2014 Public Health Nut 2018; 21(1):210-217.

6. Monteiro CA, Cannon G, Moubarac JC, Martins AP, Martins CA, Garzillo J, Canella DS, Baraldi LG, Barciotte M, Louzada ML, Levy RB, Claro RM, Jaime PC. Dietary guidelines to nourish humanity and the planet in the twenty-first century. A blueprint from Brazil. Public Health Nutr 2015; 18(13):2311-2322.

7. Davies VF, Moubarac JC, Medeiros KJ, Jaime PC. Applying a food processing-based classification system to a food guide: a qualitative analysis of the Brazilian experience. Public Health Nutr 2018; 21(1):218229.

8. Orlandi EP. Análise de Discurso: Princípios e Procedimentos. Campinas: Pontes editores; 2015.

9. Pêcheux M. L'application des concepts de la linguistique à l'amélioration des techniques d'analyse de contenu. Ethnies 1973; 3:101-118.

10. Fardet A, Rock E. Toward a new philosophy of preventive nutrition: from a reductionist to a holistic paradigm to improve nutritional recommendations. $A d v$ Nutr 2014; 5(4):430-446.

11. Fardet A, Rock E. Toward a new philosophy of preventive nutrition: from a reductionist to a holistic paradigm to improve nutritional recommendations. $A d v$ Nutr. 2014; 5(4):430-446.

12. Kraemer FB, Prado SD, Ferreira FR, Carvalho MCVS. O discurso sobre a alimentação saudável como estratégia de biopoder. Physis 2014; 24(4):1337-1360.

13. Philippi ST, Latterza AR, Cruz ATR, Ribeiro LC. Pirâmide alimentar adaptada: guia para escolha dos alimentos. Rev. Nutri. 1999; 12(1):65-80.

14. Monteiro CA, Levy RB, Claro RM, Castro IR, Cannon G. A new classification of foods based on the extent and purpose of their processing. Cad Saude Publica 2010; 26(11):2039-2049.

15. Pan American Health Organization (PAHO), World Health Organization (WHO). Ultra-Processed Food and Drink Products in Latin America: Trends, impact on obesity, policy implications. Washington: PAHO; 2015.

16. Louzada MLC, Martins APB, Canella DS, Baraldi LF, Levy R, Claro RM, Moubarac JC, Cannon G, Monteiro CA. Ultra-processed foods and the nutritional dietary profile in Brazil. Rev Saude Publica 2015; 49(38):1-11.

17. Instituto Brasileiro de Geografia e Estatística (IBGE). Pesquisa de Orçamentos Familiares 2008-2009: análise do consumo alimentar pessoal no Brasil. Rio de Janeiro: IBGE; 2011.
18. Louzada ML, Baraldi LG, Steele EM, Martins AP, Canella DS, Moubarac JC, Levy RB, Cannon G, Afshin A, Imamura F, Mozaffarian D, Monteiro CA. Consumption of ultra-processed foods and obesity in Brazilian adolescents and adults. Prev Med 2015; 81:9-15.

19. Canella DS, Levy RB, Martins AP, Claro RM, Moubarac JC, Baraldi LG, Cannon G, Monteiro CA. Ultra-processed food products and obesity in Brazilian households (2008-2009). PLoS One 2014; 9(3):e92752.

20. Thompson SV, Winham DM, Hutchins AM. Bean and rice meals reduce postprandial glycemic response in adults with type 2 diabetes: a cross-over study. Nutr J 2012; 11(1):23.

21. Mattei J, Hu FB, Campos H. A higher ratio of beans to white rice is associated with lower cardiometabolic risk factors in Costa Rican adults. Am J Clin Nutr 2011; 94(3):869-876.

22. Scrinis G. Reducing and integrating. Public Health Nutr 2006; 9:95-96.

23. Mintz SW, Du Bois CM. The Anthopology of Food and Eating. Annu. Rev. Anthropol. 2012; 31:99-119.

24. Moubarac JC, Parra DC, Cannon G, Monteiro CA Food Classification Systems Based on Food Processing: Significance and Implications for Policies and Actions: A Systematic Literature Review and Assessment. Curr Obes Rep 2014; 3(2):256-272.

25. Fischler C. Gastro-nomía y gastro-anomía. Sabiduría del cuerpo y crisis biocultural de la alimentación moderna. Gazeta de Antropología 2010; 26(1):1-19.

26. Fischler C. O futuro está na nostalgia. In: Fischler C, Masson E, organizadores. Comer. A alimentação de franceses, outros europeus e americanos. São Paulo: Editora Senac; 2010. p. 75-85.

27. Food and Agriculture Organization (FAO). FAO and traditional knowledge: the linkages with sustainability, food security and climate change impacts. Rome: FAO; 2009.

28. Oliveira MSS, Amparo-Santos L, Scagliusi FB. A abordagem da culinária no guia alimentar para a população brasileira. XII Reunión de Antropología del Mercosur. 2017 Dec 4-7; Posadas, Misiones Argentina: RAM; 2017.

29. Mills S, White M, Brown H, Wrieden W, Kwasnicka D, Halligan J, Robalino S, Adams J. Health and social determinants and outcomes of home cooking: A systematic review of observational studies. Appetite 2017; 111:116-134.

30. Engler-Stringer R. Food, cooking skills, and health: a literature review. Can J Diet Pract Re 2010; 71(3):141145.

31. Lang T, Caraher M. Is there a culinary skills transition? Data and debate from the UK about changes in cooking culture. Journal of the HEIA 2001; 8(2):1-14.

32. Growth from Knowledge (GFK). Cooking: Consumers attitudes towards, and time spent cooking. Nuremberga: GFK Market Research; 2015.

33. Dória CA. A Formação da Culinária Brasileira. São Paulo: PubliFolha; 2009.

34. Assunção V K. Comida de Mãe: notas sobre alimentação, família e gênero. Caderno Espaço Feminino 2008; 19(1):233-254 
35. Sato PM, Pereira PR, Stelmo LC, Unsain RF, Ulian MD, Sabatini F, Martins PA, Scagliusi FB. Eating practices and habitus in mothers. A Brazilian population -based survey. Appetite 2014; 82:16-28.

36. Bernardes AFM, Da Silva CG, Frutuosa MF. Alimentação saudável, cuidado e gênero: percepções de homens e mulheres da zona noroeste de Santos-SP. Demetra: Alimentação, Nutrição \& Saúde 2016; 11(3):559-573.

37. Food and Agriculture Organization (FAO), Asian Development Bank (ADB). Gender equality and food security: Women's Empowerment as a Tool Against Hunger. Philippines: ADB; 2013.

38. Jabs J, Devine CM. Time scarcity and food choices: an overview. Appetite 2006; 47(2):196-204

39. Hartmann C, Dohle S, Siegrist M. Importance of cooking skills for balanced food choices. Appetite 2013; 65:125-131.

40. Devine CM, Connors MM, Sobal J, Bisogni CA. Sandwiching it in: spillover of work onto food choices and family roles in low- and moderate-income urban households. Soc Sci Med 2003; 56(3):617-630.

41. Azeredo CM, Rezende LF, Canella DS, Claro RM, Peres MFT, Luiz OC, França-Junior I, Kinra S, Hawkesworth S, Levy RB. Food environments in schools and in the immediate vicinity are associated with unhealthy food consumption among Brazilian adolescents. Prev Med 2016; 88:73-79.

42. Dória CA. Flexionando o gênero: a subsunção do feminino no discurso moderno sobre o trabalho culinário. Cadernos PAGU. 2012; 39:251-271.

43. Monteiro CA, Moubarac JC, Cannon G, Ng SW, Popkin B. Ultra-processed products are becoming dominant in the global food system. Obes Rev. 2013; 14(Supl. 2):21-28.

44. Louzada MLDC, Ricardo CZ, Steele EM, Levy RB, Cannon G, Monteiro CA. The share of ultra-processed foods determines the overall nutritional quality of diets in Brazil. Public Health Nutr 2018; 21(1):94-102.

45. Warde A, Martens L. Eating Out: Social Differentiation, Consumption and Pleasure. Cambridge: Cambridge University Press; 2000.
46. Fischler C. Commensality, society and culture. Soc. Sci. Inf. 2011; 50(3-4):528-548.

47. Pearson N, Biddle SJ, Gorely T. Family correlates of fruit and vegetable consumption in children and adolescents: a systematic review. Public Health Nutr 2009; 12(2):267-283.

48. Videon TM, Manning CK. Influences on adolescent eating patterns: the importance of family meals. $J$ Adolesc Health 2003; 32(5):365-3373.

49. Hammons AJ, Fiese BH. Is frequency of shared family meals related to the nutritional health of children and adolescents? Pediatrics. 2011; 127(6):e1565-74.

50. Cho W, Takeda W, Oh Y, Aiba N, Lee Y. Perceptions and practices of commensality and solo-eating among Korean and Japanese university students: A cross-cultural analysis. Nutr Res Pract 2015; 9(5):523-529.

51. Phull S, Wills W, Dickingson A. Is It a Pleasure to Eat Together? Theoretical Reflections on Conviviality and the Mediterranean Diet. Sociol Compass 2015; 9(11):977-986

52. Wilk R. Power at the Table: Food Fights and Happy Meal. Cult Stud Crit Methodol 2010; 10(6):428-436.4.

53. Scagliusi FB, da Rocha Pereira P, Unsain RF, de Morais Sato P. Eating at the table, on the couch and in bed: An exploration of different locus of commensality in the discourses of Brazilian working mothers. Appetite 2016; 103:80-86.

Artigo apresentado em 28/02/2018

Aprovado em 09/10/2018

Versão final apresentada em 10/10/2018 